\title{
Announcement
}

\section{Congress of The International Society of Psychoneuroendocrinology}

The 9th Congress of The International Society of Psychoneuroendocrinology will be held in the University of Dublin, Trinity College, August 20-24, 1978.

Further information may be obtained from: Dr. Austin DARRAGH, Chairman, 9th Congress of the International Society of Psychoneuroendocrinology, IMAX, Irish Medical Association, 10, FitzwiUiam Place, Dublin 2 (Ireland). Telephone: 762087/762550/761793. 